ARTICLE

Received 19 Jul 2016 | Accepted 5 Apr 2017 | Published 30 May 2017

DOI: $10.1038 /$ ncomms15527

OPEN

\title{
Drug-seeking motivation level in male rats determines offspring susceptibility or resistance to cocaine-seeking behaviour
}

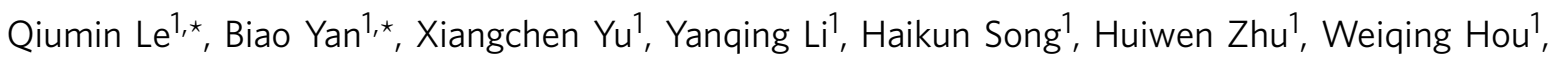
Dingailu $\mathrm{Ma}^{2}$, Feizhen $\mathrm{Wu}^{2}$, Yuqing $\mathrm{Zhou}^{1} \&$ Lan $\mathrm{Ma}^{1}$

Liability to develop drug addiction is heritable, but the precise contribution of non-Mendelian factors is not well understood. Here we separate male rats into addiction-like and non-addiction-like groups, based on their incentive motivation to seek cocaine. We find that the high incentive responding of the FO generation could be transmitted to F1 and F2 generations. Moreover, the inheritance of high incentive response to cocaine is contingent on high motivation, as it is elicited by voluntary cocaine administration, but not high intake of cocaine itself. We also find DNA methylation differences between sperm of addiction-like and non-addiction-like groups that were maintained from FO to F1, providing an epigenetic link to transcriptomic changes of addiction-related signalling pathways in the nucleus accumbens of offspring. Our data suggest that highly motivated drug seeking experience may increase vulnerability and/or reduce resistance to drug addiction in descendants.

\footnotetext{
${ }^{1}$ Department of Neurosurgery, and Institute of Translational Neuroscience, Huashan Hospital, State Key Laboratory of Medical Neurobiology, School of Basic Medical Sciences and Institutes of Brain Science, Fudan University, 138 Yixueyuan Road, Shanghai 200032, China. ${ }^{2}$ Laboratory of Epigenetics, Institutes of Biomedical Sciences, Fudan University, 138 Yixueyuan Road, Shanghai 200032, China. *These authors contributed equally to this work. Correspondence and requests for materials should be addressed to L.M. (email: lanma@fudan.edu.cn).
} 
A cquisition of nutritional resources is one of the critical driving forces in evolution ${ }^{1}$. Pursuit of food and water, which produces positive effects on the reward system, are reinforced and inherited as natural rewards. Dynamic interactions between genes and experiences are involved in the selective adaptation to new potential rewarding stimuli during evolution ${ }^{2}$. Emerging evidence indicates that, in addition to natural selection of genetic variants, non-Mendelian epigenetic changes induced by ancestral environmental and behavioural perturbations also contribute to the transgenerational inheritance of adaptive phenotypes ${ }^{3-6}$.

Addictive substances of abuse, such as cocaine and heroin, induce much stronger rewarding effects than natural rewards. Chronic consumption of such drugs can lead to addiction, which is characterized by an increase in consumption of drugs with enhanced motivation at the expense of individual's health and social life ${ }^{7,8}$. The major substrates that cause persistent compulsive drug use are attributed to molecular and cellular adaptations that occur in the mesolimbic system, which largely overlaps with the nature reward circuit ${ }^{9}$.

Indeed, epidemiological studies show a remarkable degree of familial aggregation of substance abuse ${ }^{10,11}$. Large-scale twin and adoption studies have demonstrated that the heritability of liability for substance abuse is between 50 and 60\% (refs 12-15). The high heritability has been largely attributed to, but not fully explained by, variations in the genome ${ }^{16}$. We thus hypothesized that being addicted to $\operatorname{drug}(\mathrm{s})$ of abuse may promote epigenetic adaptation in favour of a positive responding (for example, enhanced motivation to seek drug reinforcement) to the drug reward, resulting in an increased vulnerability to addiction in subsequent generations.

Epidemiological studies show that $<20 \%$ of drug users become addicted $^{14,17}$, which was confirmed by laboratory animal experiments $^{18}$. Here in an attempt to explore the effect of cocaine-induced reinforcement in subsequent generations, we divide F0 rats into two groups by their incentive responding to cocaine reward. We show that F1 and F2 offspring sired by rats with prior experience of high motivational self-administration of cocaine (which we term Addict rats) exhibit increased susceptibility or reduced resistance to cocaine-seeking behaviour, and that this transgenerational effect is dependent on high incentive motivation induced by cocaine, but not high intake of cocaine per se. Reduced representation bisulfite sequencing reveal persistent changes in DNA methylation of sperm DNA in F0 and F1 of rats displaying addiction-like behaviour, suggesting possible involvement of epigenetic mechanisms in this behavioural effect.

\section{Results}

Cocaine reinforcement is transgenerationally inherited. To explore the potential transgenerational effects of cocaine-induced reinforcement, naive male Sprague-Dawley rats were allowed to self-administer cocaine $\left(0.75 \mathrm{mg} \mathrm{kg}^{-1}\right.$ per injection, Cocaine-SA group) or saline (Saline-SA group) by pressing the active lever in 4 -h daily sessions. Rats were first subjected to self-administration at a fixed ratio (FR) of 1 (that is, one lever press for an injection), then switched to FR3 and FR5 (three and five lever presses for one injection, respectively) on days 6 and 8 , and finally tested on a progressive ratio (PR) schedule on day 13 to assess their motivation for drug reinforcement. Compared with Saline-SA group, Cocaine-SA rats showed significantly higher lever presses during the FR schedules (Fig. 1a left) and higher break point (the number of lever presses to earn the last cocaine injection) in the PR test (Fig. 1a right).

The individual performance of each of the 134 Cocaine-SA rats in cocaine self-administration was analysed. Uncontrollable urges to seek drugs and excessive drug consumption are important characteristics of addiction. Thus, to reflect the incentive motivational responding to cocaine reward, a combined behavioural score was calculated for each Cocaine-SA rat, by addition of normalized score derived from average lever pressing during FR5 program, and break point during PR schedule. Cocaine-SA rats with the top $25 \%$ and the bottom $40 \%$ of the behavioural score were designated as Addict F0 (red circle) and Non-addict F0 (blue circle), respectively (Fig. 1b).

The Addict and Non-addict (F0) rats were then mated with naive female rats (Fig. 1c). Addict and Non-addict F1 rats (17 and 16 litters respectively) were obtained, and 2-6 adult male rats from each litter were subjected to the cocaine self-administration test. The F1 offspring of Addict rats, as compared with Nonaddict F1, exhibited higher lever presses for cocaine injections during the FR program, and higher motivation for cocaine reinforcement as indicated by break point (Fig. 1d). Interestingly, the third litter of Addict F0, conceived after 6-7 weeks of abstinence, still showed higher voluntary cocaine intake in FR5 sessions compared with the third litter from Non-addict rats (Supplementary Fig. 1). Hyperactivity, reduced cocaine sensitivity and enhanced reward learning ability may increase lever presses in self-administration. Therefore, we conducted three series of experiments to exclude the possibility that any of the abovementioned factors might have influenced our behavioural readout. In the open field test, no difference in basal locomotor activity and anxiety level between Addict and Non-addict F1 rats was observed (Fig. 2a). Moreover, Addict F1 and Non-addict F1 showed similar dose-dependent responses to cocaine in the sensitivity test (Fig. 2b). Finally, in sucrose and food (natural rewards) self-administration tests, the two groups exhibited equivalent self-administration learning curves and break points (Figs 1e and 2c).

The phenotype observed in F1 generation could arise from direct exposure of the sperm of F0 rats to cocaine rather than from transgenerational transmission mechanisms. To exclude this possibility, Addict F2 and Non-addict F2 rats, sired by naive F1, were subjected to evaluations in cocaine self-administration. Similar to our observations in their F0 and F1 ascendants, Addict F2 rats displayed higher lever presses under FR5 schedule, and higher break point than Non-addict F2 (Fig. 3a), while their performance in sucrose self-administration (Fig. 3b), open field (Fig. 3c), and their response to acute cocaine doses was comparable to Non-addict F2 group (Fig. 3d). These data suggest that paternal propensity to develop high cocaine-seeking behaviour could be transgenerationally maintained in subsequent generations.

Motivation determines cocaine response in offspring. An alternative interpretation for the transgenerational effect is that Addict F0 self-administered more cocaine than Non-addict F0. Thus, inheritance could stem from the exposure to cocaine per se and may not represent incentive motivational effect of cocaine. To examine the effect of cocaine exposure, another cohort of naive rats was given twice-daily intraperitoneal injections of cocaine $\left(15 \mathrm{mg} \mathrm{kg}^{-1}\right.$, Cocaine-IP) or saline (Saline-IP) for 7 days and the response of their F1, F2 offspring in cocaine selfadministration was tested (Fig. 4a). Interestingly, Cocaine-IP F1 sired by rats receiving non-contingent cocaine injections showed significantly lower responses than Saline-IP F1 rats (Fig. 4b), while the F2 offspring of Cocaine-IP and Saline-IP exhibited no difference in cocaine self-administration (Fig. 4c). Moreover, as shown in Supplementary Fig. 2, cocaine response of Addict F1 group was significantly higher than Saline F1 under FR5 schedule, and Non-addict F1 exhibited lower breakpoint than Saline F1. These data suggest that chronic cocaine intake 
a
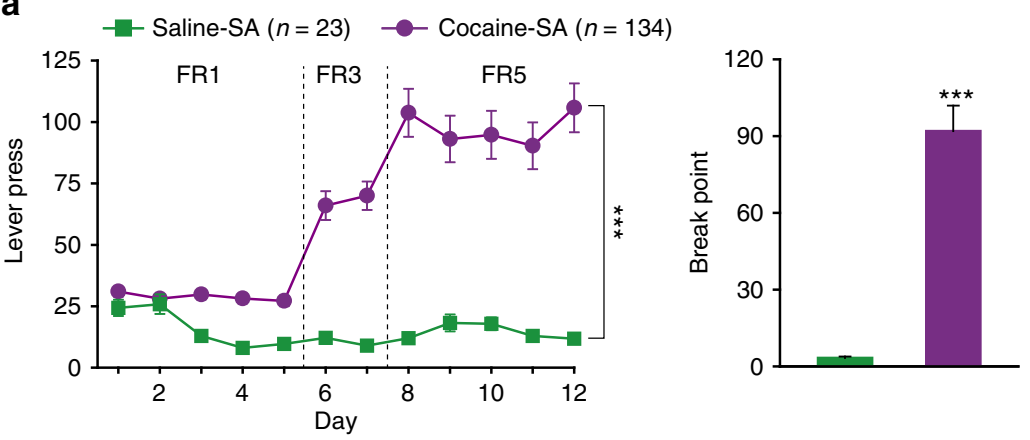

b

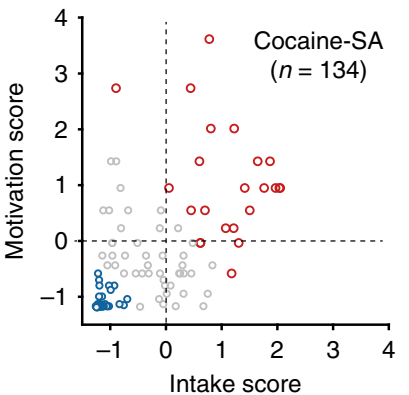

C

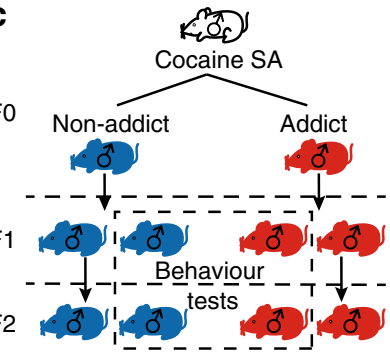

d

Cocaine SA
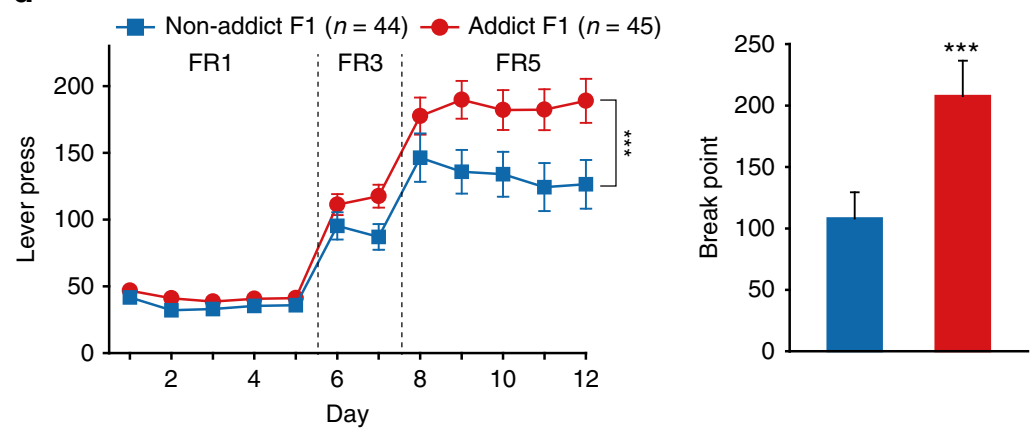

e

Sucrose SA
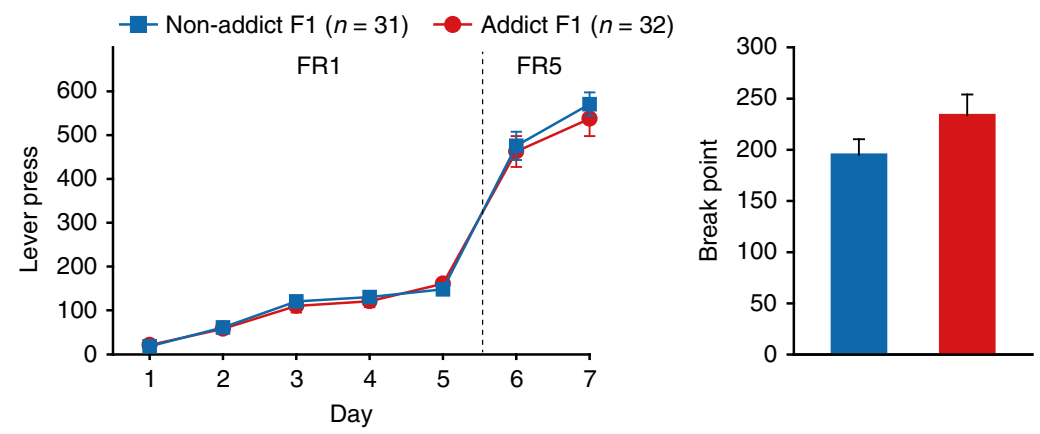

Figure 1 | Performance of Addict and Non-addict FO rats in cocaine self-administration. (a) Naive rats were trained to self-administer saline (Saline SA) or cocaine (Cocaine SA) under a 5-day FR1, 2-day FR3, 5-day FR5, 1-day PR schedule. Cocaine-SA group showed higher lever presses at FR (left) and break point (right) at PR schedules, compared with Saline-SA group. Lever press, Group $\times$ FR, mixed model repeated measures $(M M R M), \chi^{2}(2)=101.91$, $P<0.001$; Group, ${ }^{\star \star \star} P<0.001$, Saline-SA versus Cocaine-SA. Break point, Mann-Whitney Rank-Sum Test, $Z=4.688,{ }^{\star \star \star} P<0.001$. Saline-SA, $n=23$; Cocaine-SA, $n=134$. (b) Scores for cocaine intake and motivation of each Cocaine-SA rat were calculated according to SA performance at FR5 and PR schedules, respectively, and plotted. Cocaine SA rats with a combined drug-seeking behavioural score within the top $25 \%$ and the bottom $40 \%$ were designated as Addict FO (red circle) and Non-addict FO (blue circle), respectively. (c) Addict FO and Non-addict FO rats were crossed with naive female rats to generate Addict F1 and Non-addict F1, which were then randomly designated for mating to generate F2 or for behavioural testing. (d) Addict F1 rats displayed higher cocaine consumption and motivation than Non-addict F1. Lever press, Group $\times$ FR, MMRM, $\chi^{2}(2)=23.33, P<0.001$; Group, $P=0.041$; FR5, ${ }^{\star \star \star} P<0.001$, Non-addict F1 versus Addict F1. Break point, Mann-Whitney Rank-Sum Test, $Z=3.272$, ${ }^{\star \star \star} P<0.001$. Non-addict F1, $n=44 ;$ Addict F1, $n=45$. (e) There was no difference in intake and motivation for sucrose reward in F1. Lever press, Group $\times$ FR, MMRM, $\chi^{2}(1)=10.1, P=0.32$. Break point, Mann-Whitney Rank-Sum Test, $Z=1.772, P=0.076$. Non-addict F1, $n=31$; Addict F1, $n=32$. Data are expressed as mean \pm s.e.m. 

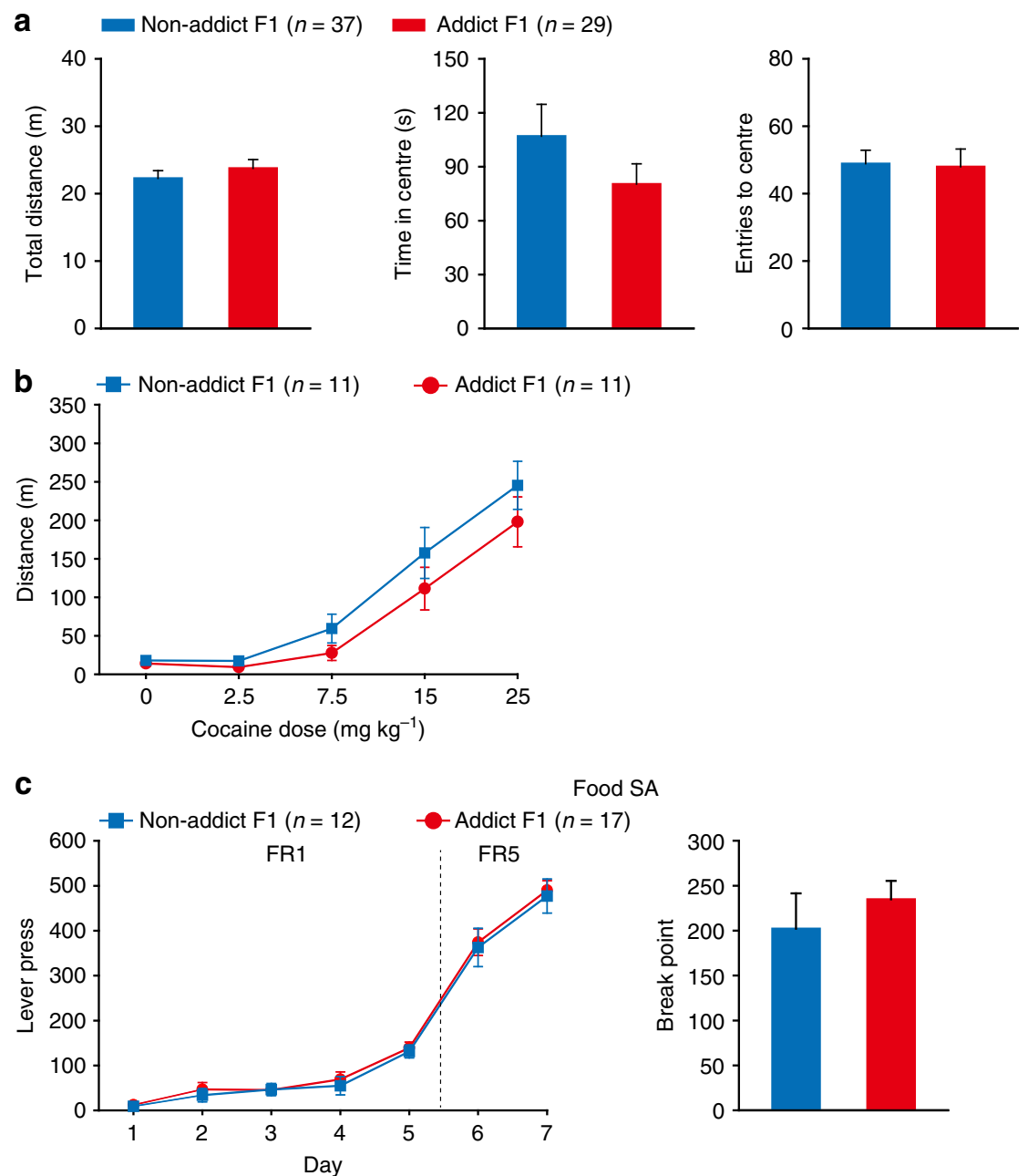

Figure 2 | Comparable performance of Addict F1 and Non-addict F1 rats in basal and cocaine-induced locomotion activity and food self-administration. (a) Open field test. Total distance, Student's $t$-test, $t(64)=0.872, P=0.386$. Time in centre, Student's $t$-test, $t(64)=1.197, P=0.236$. Entries to centre, Student's $t$-test, $t(64)=0.142, P=0.887$. Non-addict $F 1, n=37$; Addict $F 1, n=29$. (b) Sensitivity to cocaine-induced locomotor activity. Two-way RM ANOVA, $F_{\text {Group } \times \text { Dose }}(4,80)=0.734, P=0.571$. Non-addict F1, $n=11$; Addict F1, $n=11$. (c) Food self-administration test. Lever press, Group $\times F R$, MMRM, $\chi^{2}(1)=0.19, P=0.663$. Break point, Mann-Whitney Rank-Sum Test, $Z=1.039, P=0.299$. Non-addict F1, $n=12 ; A d d i c t F 1, n=17$. Data are expressed as mean \pm s.e.m.

results in lower drug responding in F1 generation, that is, a protective effect, and that the high response for cocaine-induced reinforcement observed in Addict offspring is unlikely caused solely by excessive exposure to high doses of cocaine. Previous studies have shown that only a portion of drug users or animals subjected to self-administration of addictive drugs become addicted $^{17,18}$. Combined with the result of Vassoler et al. ${ }^{19}$ showing that F1 offspring of rats that self-administered cocaine exhibit lower responding than F1 from saline exposed animals, we speculate that the transgenerational transmission of high cocaine incentive response observed may be dependent on the addictionlike state in the F0 generation, and the incentive motivational effect causes increased susceptibility to cocaine-seeking behaviour or decreased protective effect in offspring.

To further discriminate the effects of non-contingent and motivational intake of cocaine, yoked cocaine infusion experiment was carried out. In yoked cocaine delivery procedure, one rat self-administered cocaine by actively pressing the lever (SA), while the paired rat (Yoke) passively received the same dose of cocaine at the same time (Fig. 5a). Addict and Non-addict F0 in $\mathrm{SA}$, as determined by the combined score, and their paired Yoke groups, were mated to generate F1. In contrast to the significantly higher drug intake and motivation for drug reinforcement observed in F1 of Addict-SA group compared with Non-addictSA group, no difference in lever presses or break point for cocaine was observed between Addict-Yoke F1 and Non-addict-Yoke F1 rats (Fig. 5b). Non-addict-SA F1, but not Addict-SA F1, exhibited lower FR5 responding and break point than Saline-SA F1 rats (Fig. 5b), suggesting a loss of a protective effect in the Addict-SA F1 group. Each pair of Addict-SA and Addict-Yoke F0 rats received the same amount of cocaine simultaneously; however, the offspring of the two groups exhibited significant difference in lever press for cocaine. There was no difference in cocaine selfadministration found between offspring of Saline-SA and SalineYoke groups. These results support the notion that high incentive motivation for cocaine, but not the intake of an addictive drug itself, renders transgenerational inheritance of vulnerability and/ or reduce resistance to drug addiction in descendants.

As Vassoler et al. ${ }^{19}$ found that the cocaine-resistant phenotype was attributed to increase in BDNF level of F1 male cocaine sired rats, we also measured BDNF level in mPFC of drug- and experiment- naive F1 rats from Saline, Non-addict-SA, Nonaddict-Yoke, Addict-SA and Addict-Yoke groups (Fig. 6). The results showed that, F1 descendants of cocaine-experienced 
a
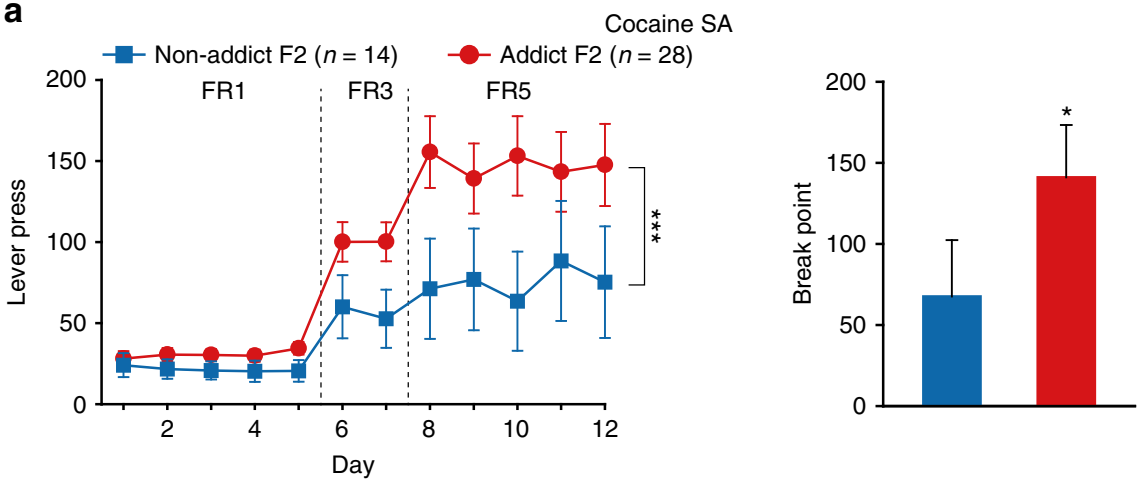

b Sucrose SA

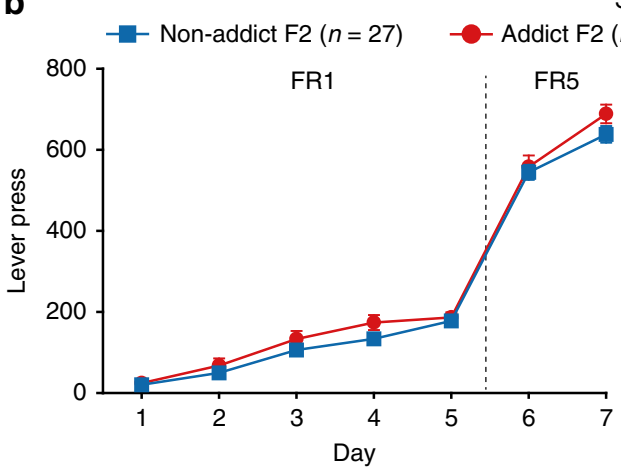

$(n=21)$

C

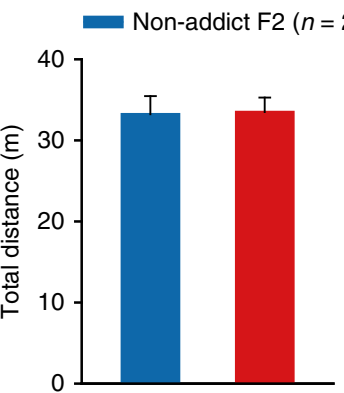

$$
\text { Addict F2 }(n=33)
$$
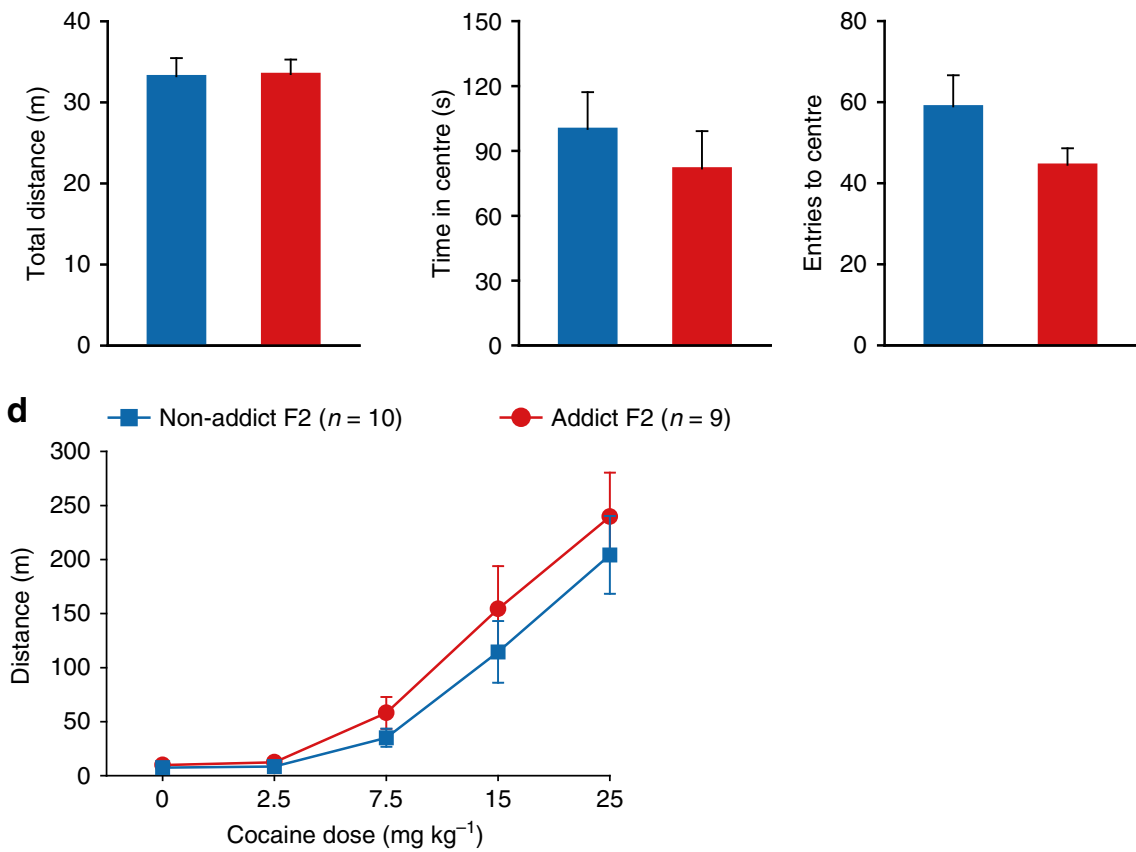

Figure 3 | Addict F2 displayed higher responding to cocaine-induced reinforcement than Non-addict F2. (a) Addict F2 rats displayed higher cocaine consumption and motivation than Non-addict F2. Lever press, Group $\times$ FR, MMRM, $\chi^{2}(2)=28.63, P<0.001$; Group, $P=0.052$; FR5, *** $P<0.001$, Nonaddict F2 versus Addict F2. Break point, Mann-Whitney Rank-Sum Test, $Z=2.513,{ }^{\star} P=0.013$. Non-addict F2, $n=14 ; A d d i c t ~ F 2, n=28$. (b) Sucrose selfadministration. Lever press, Group $\times F R, M M R M, \chi^{2}(1)=0.29, P=0.588$. Break point, Mann-Whitney Rank-Sum Test, $Z=0.783, P=0.433$. Non-addict $F 2, n=27$; Addict F2, $n=21$. (c) Open field test. Total distance, Student's $t$-test, $t(54)=0.0964, P=0.924$. Time in centre, Student's $t$-test, $t$ (54) $=0.729$, $P=0.469$. Entries to centre, Student's $t$-test, $t(54)=-1.796, P=0.0781$. Non-addict F2, $n=23$; Addict F2, $n=33$. (d) Cocaine-induced locomotor activity. Two-way RM ANOVA, $F_{\text {group } \times \text { day }}(4,68)=1.170, P=0.295$. Non-addict F2, $n=10$; Addict F2, $n=9$. Data are expressed as mean \pm s.e.m.

fathers (Non-addict-SA, Non-addict-Yoke, Addict-SA, and Addict-Yoke rats) showed cocaine dose-dependent elevation of BDNF level, compared with F1 offspring of Saline-SA group. However, BDNF level between SA and yoked rats from Addict or
Non-addict groups was not different. These data indicate that paternal cocaine experience induces BDNF elevation in F1 offspring, and this is correlated with the amount of cocaine taken by F0 fathers, not motivation for reinforcement. 

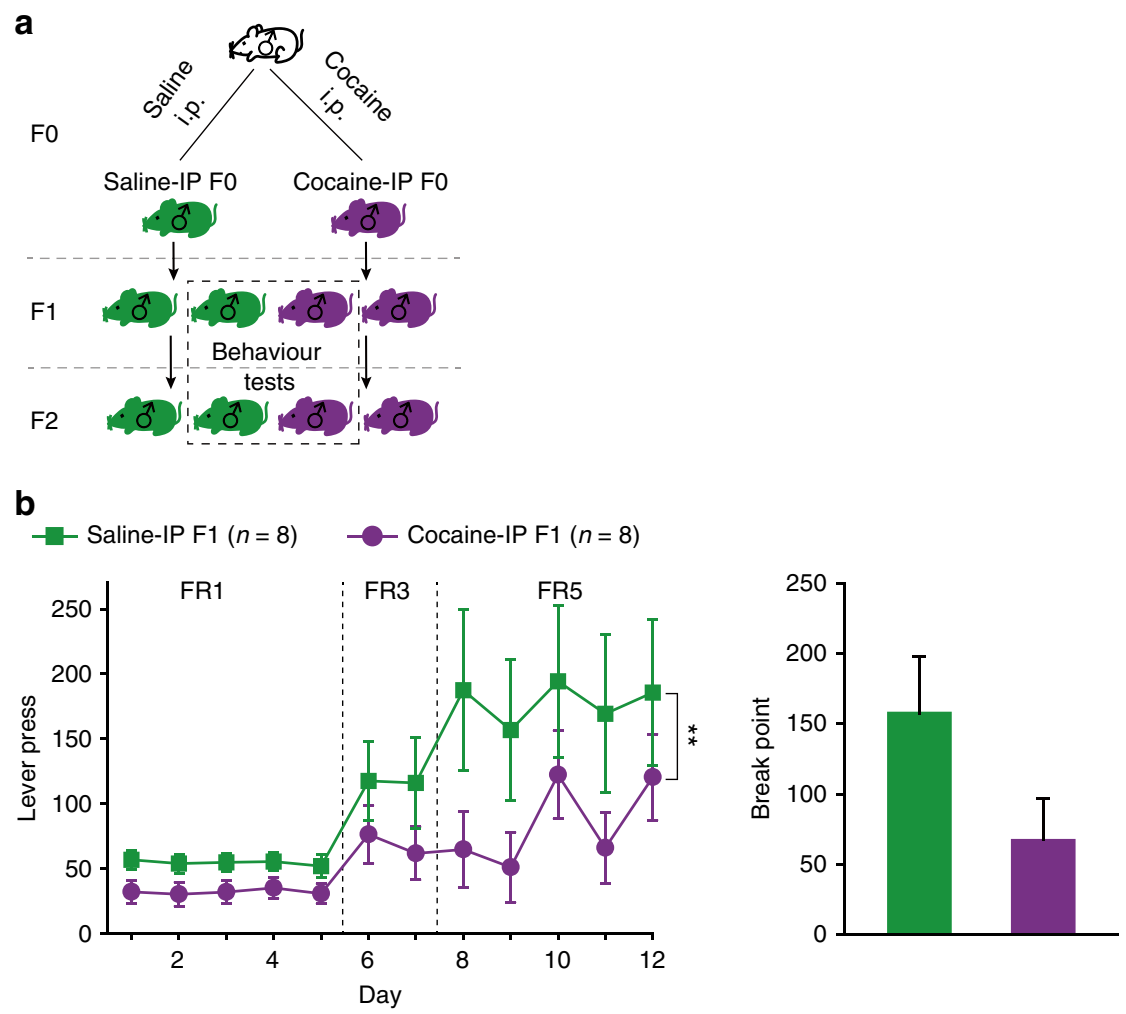

C
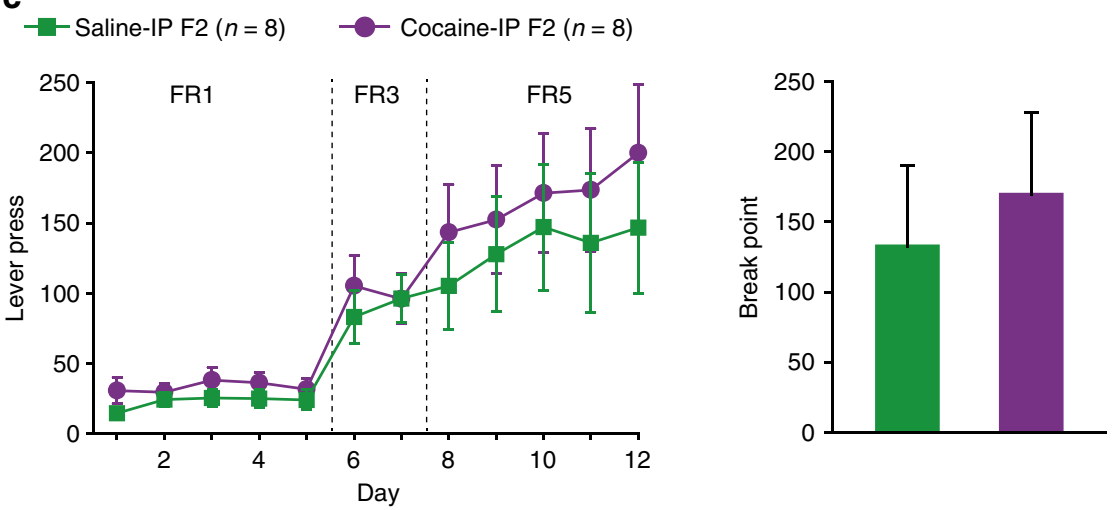

Figure 4 | Non-contingent chronic cocaine injections induce inter-generational cocaine resistance. (a) Rats received $15 \mathrm{mg} \mathrm{kg}{ }^{-1}$ cocaine (Cocaine-IP FO) or equal volume of saline (Saline-IP FO) intraperitoneal injections twice daily for 7 days and were mated with naive females one day after the last injection. Four litters from each of group were randomly designated for behavioural experiment or for mating to generate F2. (b) Cocaine-IP F1 did not show an increase but displayed a decrease tendency in lever press and break point for cocaine in self-administration test, compared with Saline-IP F1 rats. Lever press, Group $\times$ FR, MMRM, $\chi^{2}(2)=13.00, P=0.0015 ; F R 5,{ }^{\star \star} P=0.009$, Saline-IP F1 versus Cocaine-IP F1. Break point, Mann-Whitney Rank-Sum Test, $Z=1.060, P=0.289$. Saline-IP F1, $n=8$; Cocaine-IP F1, $n=8$. (c) There was no statistical difference in lever press or break point between Saline-IP F2 and Cocaine-IP F2. Lever press, Group $\times$ FR, MMRM, $\chi^{2}(2)=2.60, P=0.272$. Break point, Mann-Whitney Rank-Sum Test, $Z=0.638, P=0.526$. Saline-IP F2, $n=8$; Cocaine-IP F2, $n=8$.

Inheritance of drug seeking behaviour is an acquired trait. The transgenerational transmission of high incentive motivation for cocaine could be attributed to pre-existing genetic variations or to acquired heritable epigenetic modifications. To dissociate the two possible mechanisms, we mated naive rats first to generate F1 offspring, and one week after mating, subjected the mated males to cocaine self-administration to identify Addict and Non-addict rats according to their performance (Fig. 7a). The offspring sired by Addict and Non-addict F0 rats before their exposure to cocaine were designated as Pre-Addict F1 and Pre-Non-addict F1 rats. In contrast to what we have observed in descendants of F0 rats mated after drug exposure (Fig. 1d), Pre-Addict F1 and Pre-Non-addict F1 rats exhibited no difference in cocaine self-administered or motivation for cocaine in PR test (Fig. 7b). No differences were observed in the animals' performance in the open field or the sucrose acquisition and motivation between these two groups (Supplementary Figs. 3a,b). Moreover, cocaine intake and motivation were similar in the mated and non-mated rats (Supplementary Fig. 3c). These data, combined with the results of yoke and intraperitoneal administration experiments, suggest that transgenerational inheritance of high incentive motivation for cocaine is an acquired trait that involves nongenetic mechanisms.

Sperm methylome difference is maintained across generations. DNA methylation, a stable epigenetic modification that regulates 
a
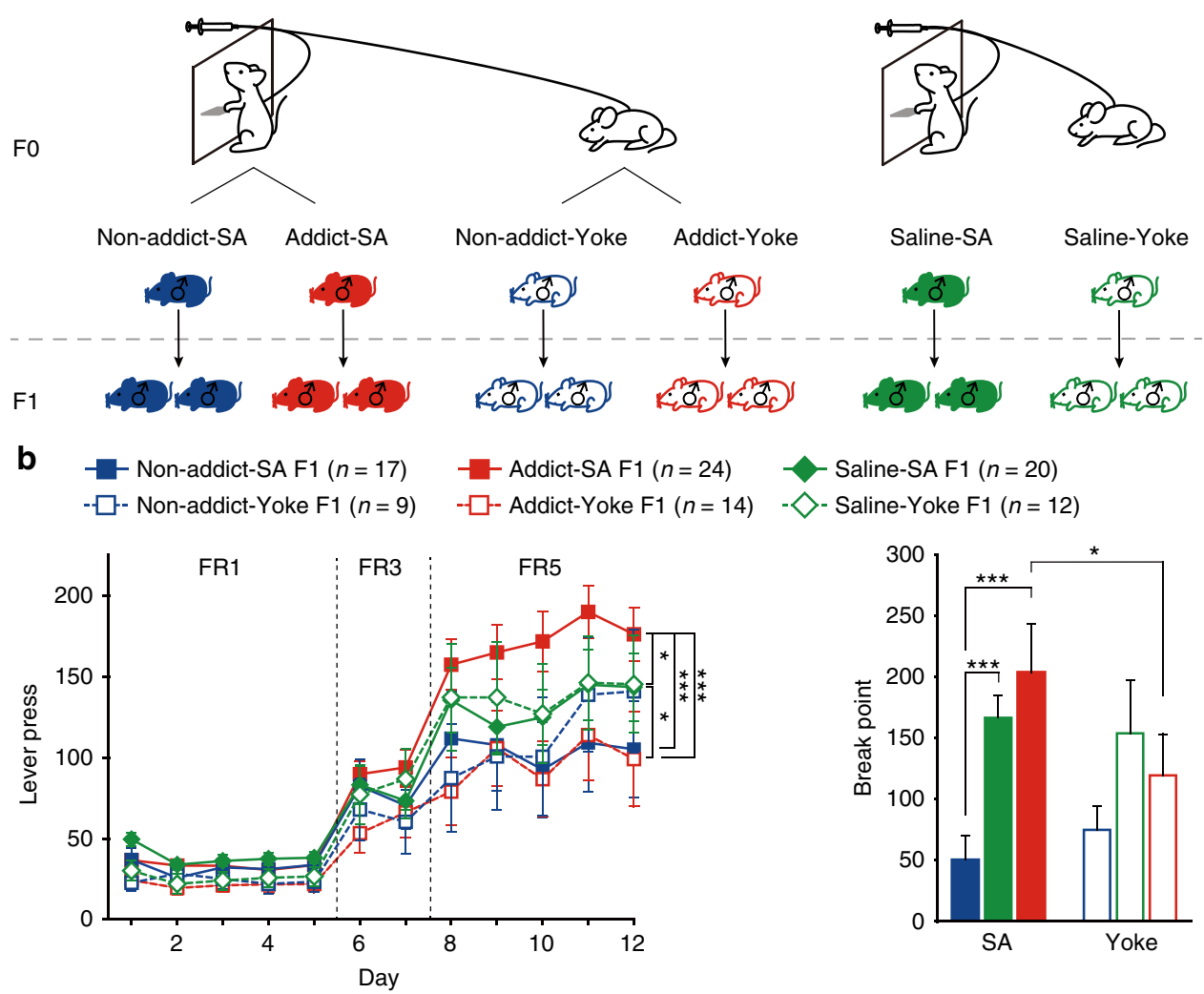

Figure 5 | The inheritance of vulnerability to cocaine reinforcement is contingent on highly motivated cocaine self-administration. (a) Naive male rats were randomly paired. In each pair, one rat was allowed to actively self-administer cocaine or saline by pressing the lever (SA group), and with each drug infusion by SA group rat, a yoked infusion of exactly the same dose of cocaine or saline (Saline-Yoke) was delivered at the same time passively (without lever press) to the paired rat (Yoke group). The yoked rats corresponding to Saline-SA, Addict-SA and Non-addict-SA groups were designated as SalineYoke, Addict-Yoke and Non-addict-Yoke, respectively. (b) Addict-SA F1 rats showed significantly higher lever press and break point for cocaine than Nonaddict-SA F1, but there was no difference between Addict-Yoke F1 and Non-addict-Yoke F1. Lever press, Group $\times$ Yoke $\times$ FR, MMRM, $\chi^{2}(4)=35.55$, $P<0.001 ; P<0.001$, Saline F1 versus Non-addict F1, $P<0.001$, Non-addict F1 versus Addict F1; FR5, ${ }^{\star \star \star} P<0.001$, Addict-SA F1 versus Non-addict-SA F1, ${ }^{\star} P=0.013$, Addict-SA F1 versus Saline-SA F1, ${ }^{\star \star \star}{ }^{*} P<0.001$, Addict-SA F1 versus Addict-Yoke F1; ${ }^{*} P=0.038$, Addict-Yoke F1 versus Saline-Yoke F1. Break point, two-way ANOVA by Ranks, $F_{\text {Group } \times \text { Yoke }}(2,91)=3.604, P=0.090$; ${ }^{\star \star \star} P<0.001$, Addict-SA F1 versus Non-addict-SA F1, ${ }^{\star \star \star} P<0.001$, Non-addict-SA F1 versus Saline-SA F1, ${ }^{\star} P=0.041$, Addict-SA F1 versus Addict-Yoke F1. Non-addict-SA F1, $n=17$; Non-addict-Yoke F1, $n=9$; Addict-SA F1, $n=24 ;$ AddictYoke F1, $n=14$; Saline-SA F1, $n=20$; Saline-Yoke F1, $n=12$. Data are expressed as mean \pm s.e.m.

gene transcription and splicing, may be one of possible mechanisms for transgenerational inheritance of acquired traits ${ }^{20}$. We therefore performed reduced-representation bisulfite sequencing (RRBS) to assess DNA methylation-enriched sites in sperm samples from Saline F0, Addict F0, Non-addict F0, Addict F1, and Non-addict F1. Sperm samples of 3-4 rats per group were individually sequenced at 20M-read depth. In the 16 samples sequenced, total $1,570,110 \mathrm{CpGs}$ were detected in more than 6 samples and with $\geq 5 \times$ coverage. We first calculated the associations of generation (F0,F1) and phenotype (Non-addict, Addict, Saline) traits with the global methylation profiles. Moderate general demethylation across all genetic elements analysed was detected in both Addict F0 and Non-addict F0 sperms, compared with Saline F0, likely due to the effect of cocaine intake; and there was also difference between Addict and Non-addict groups, indicating possible association of DNA methylation with incentive motivation for cocaine (Fig. 8a).

Multidimensional scaling revealed clear segregation of F0 with F1 generations in Dimension 1, correlating with the global demethylation caused by direct drug exposure, and separation of Addict and Non-addict groups in Dimension 2 (Fig. 8b), Notably, Fisher's exact test also indicate associations of generation and phenotype traits (generation, $P=4.0 \times 10^{-5}$; phenotype, $P=4.0 \times 10^{-5}$; generation $\times$ phenotype, $P=0.015$ ).

Next, we sought to find the methylation differences, if any, that might be relevant to the transgenerational inheritance. Therefore, we identified $\mathrm{CpG}$ sites that were differentially methylated between Addict versus Non-addict F0 and maintained across generations. We found that methylation at 1,244 $\mathrm{CpG}$ sites was significantly different between Addict and Non-addict F0 (Fig. 4c, upper panel, $P<0.05)$ and $522 \mathrm{CpG}$ sites were differentially methylated in their F1 offspring (Fig. 8c, middle panel). Importantly, 475 differentially methylated $\mathrm{CpG}$ sites were maintained in F0 and F1 generation (hereafter referred to as Maintained Differences, Fig. 8c, lower panel). Maintained methylation differences were enriched in hypo-methylated regions (maximum methylation level $\leq 0.3,93.7 \%$ in CpGs with Maintained Differences versus $34.2 \%$ in CpGs commonly covered) and also featured in TSS $\pm 2,000 \mathrm{bp}$ distribution (Fig. 8d; 66.5\% in CpGs with Maintained Differences versus $34.8 \%$ in CpGs commonly covered), indicating their potential involvement in transcriptional modulation of promoter region. Ontology analysis of genes with Maintained Differences in $\mathrm{TSS} \pm 2,000 \mathrm{bp}$ region also indicate that GO terms associated 


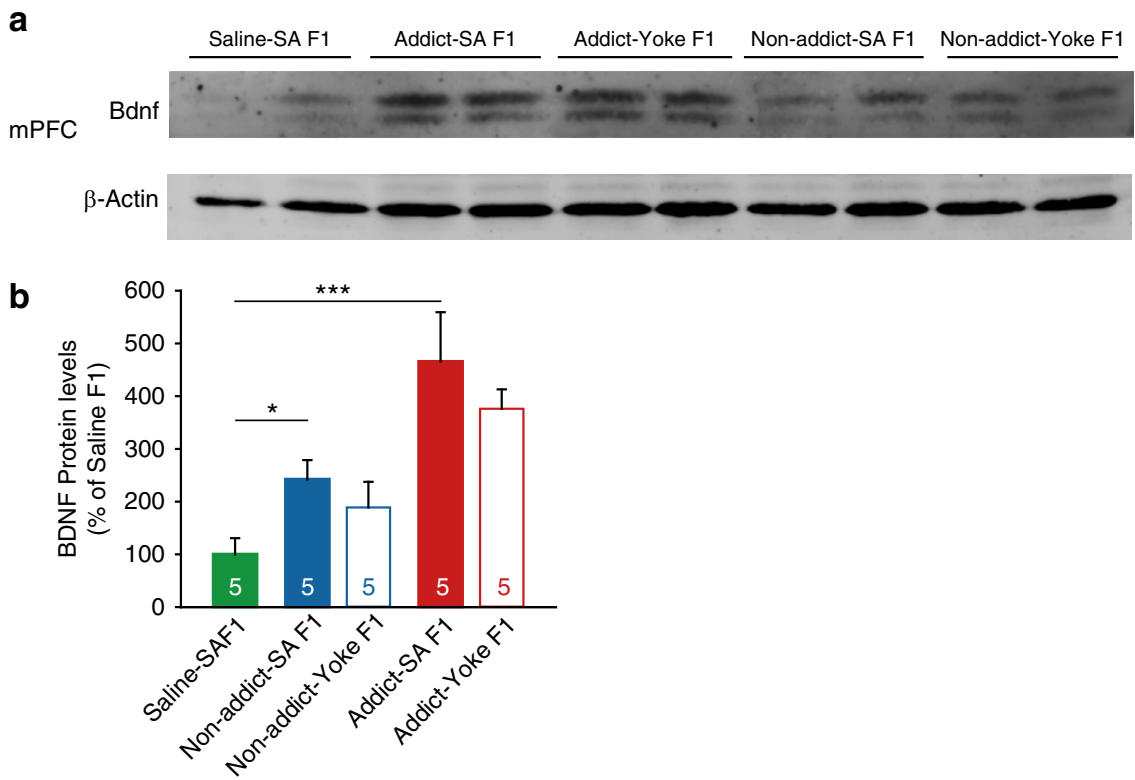

Figure 6 | Elevated BDNF level in F1 correlates with the amount of cocaine taken by F0 fathers. BDNF level in mPFC of cocaine naive F1 rats of Saline-SA, Non-addict-SA, Non-addict-Yoke, Addict-SA and Addict-Yoke groups. (a) Representative blotting of two samples from each group was shown.

(b) Statistical analysis of BDNF level in each group. BDNF level normalized by $\beta$-actin was acquired, and then relative protein level of each sample was divided by average of BDNF/ $\beta$-actin in Saline F1 group. One-way ANOVA, $F(4,20)=7.251, P<0.001$; Bonferroni post hoc, ${ }^{\star \star \star} P<0.001$, compared with Saline F1. $n=5$ each. Data are expressed as mean \pm s.e.m.
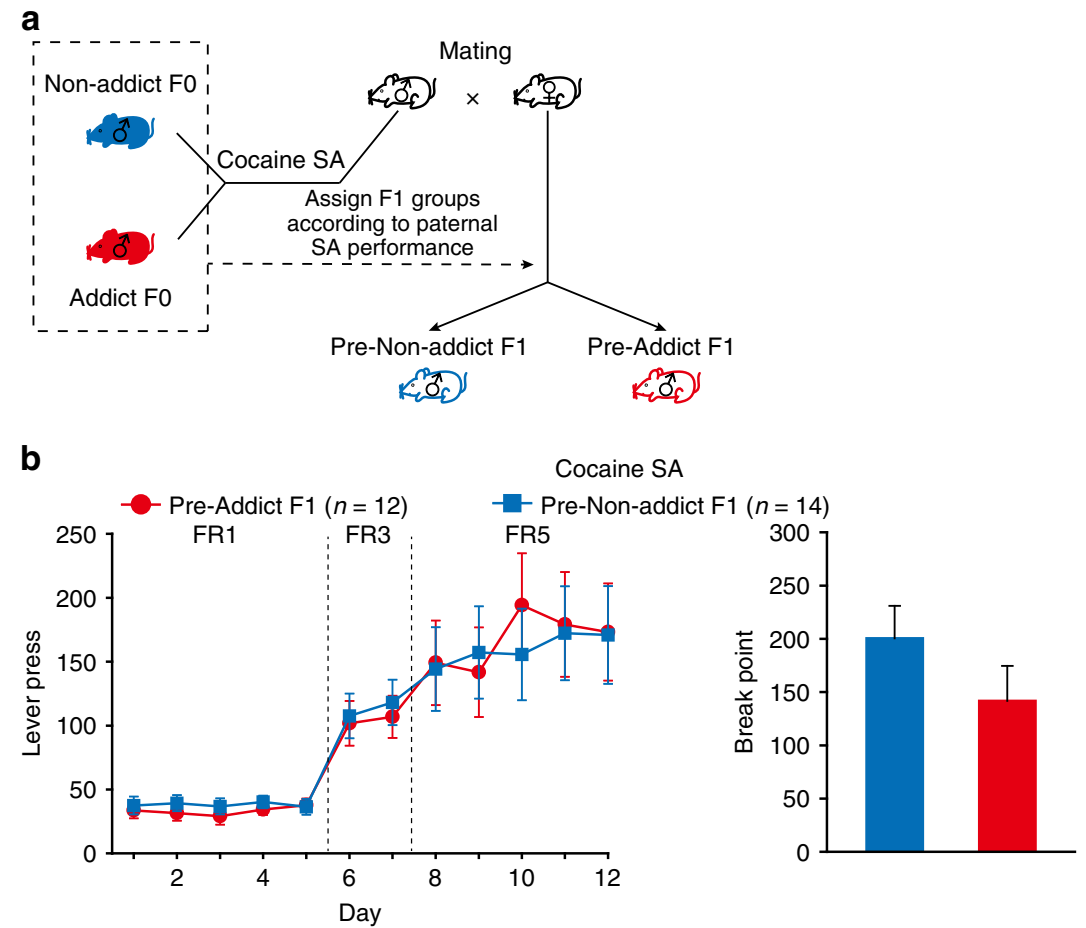

Figure 7 | Transgenerational inheritance of cocaine reinforcement is an acquired trait. (a) Naive male rats were crossed with naive females first, and one week after mating were subjected to cocaine self-administration procedures to identify Addict and Non-addict rats according to their cocaine intake and motivation scores. The offspring sired by Addict and Non-addict rats (before their exposure to cocaine) were designated as Pre-Addict F1 and Pre- Nonaddict F1. (b) Pre- Non-addict F1 and Pre-Addict F1 rats showed no difference in lever press and break point. Lever press, Group $\times$ FR, MMRM, $\chi^{2}(2)=0.79, P=0.68$. Break point, Mann-Whitney Rank-Sum Test, $Z=1.273, P=0.203$. Pre- Non-addict F1, $n=14 ;$ Pre-Addict F1, $n=12$. Data are expressed as mean \pm s.e.m.

with development and morphology are overrepresented (Fig. 8e and Supplementary Table 1). Interestingly, binding motifs for transcription factors, including KLF4, SP1, EGR1 and ZFX, were enriched in these regions (Fig. 8f). Transcription activity of KLF4, which has the most significant e-value, has been demonstrated to be regulated by methylation level ${ }^{21}$. KLF4 binding sites were detected in promoters of Btg2 and $\mathrm{Nr} 4 \mathrm{al}$, and they exhibited preserved higher methylation in Addict groups than Non-addict 
a

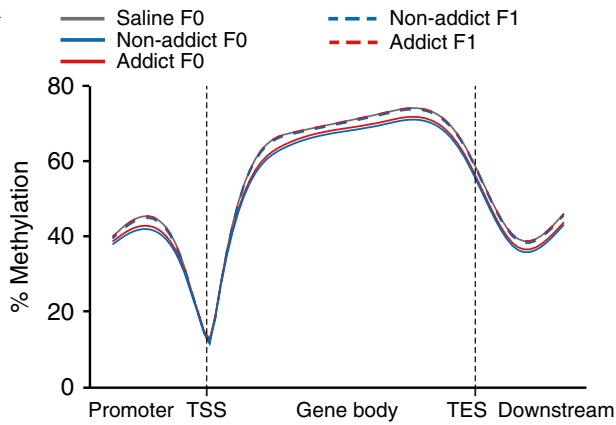

b

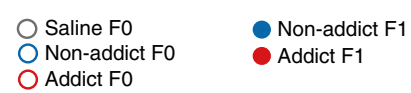

$\mathrm{O}$ Addict FO

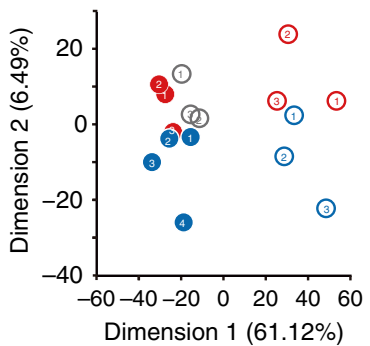

C

Differentially methylated $\mathrm{CpG}$ count

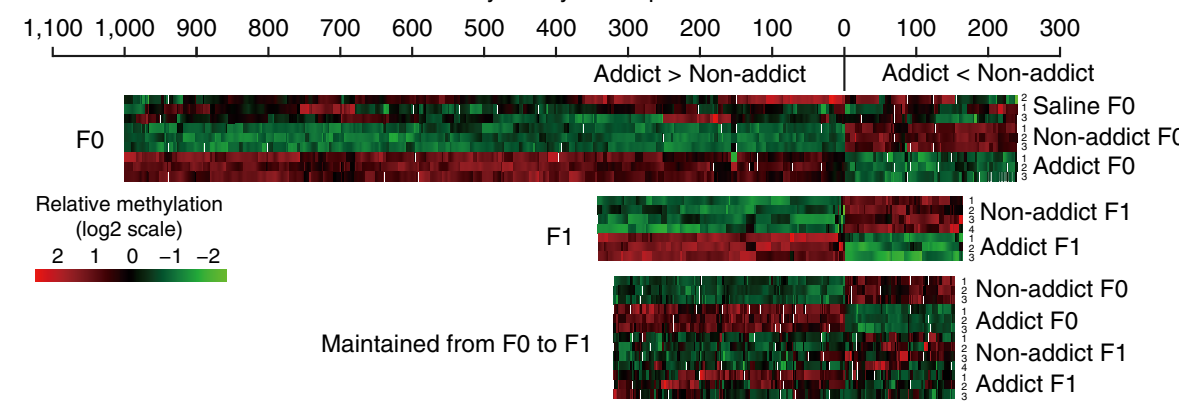

d

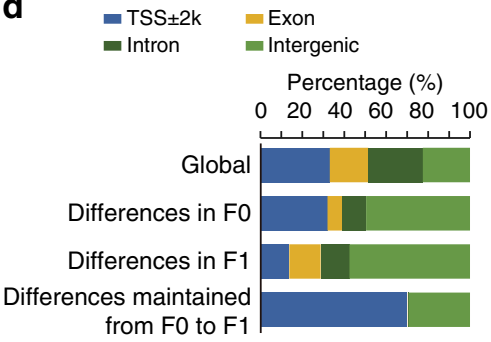

f

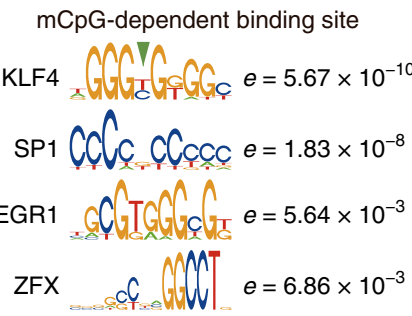

g e

GO terms

$Z$ score

$-2.3-2.4-2.5-2.6-2.7$

Regulation of organ formation Acrosome assembly AMP metabolic process

$T$ cell differentiation in thymus Histone $\mathrm{H} 2 \mathrm{~A}$ monoubiquitination Positive regulation of poly $(A)$ shortening Ephrin receptor signalling pathway Cellular component morphogenesis Neural crest cell migration Pattern specification process

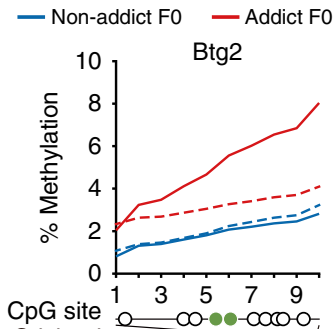

CpG island Gene structure

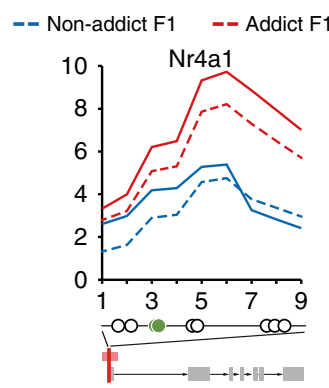

Figure 8 | Identification of maintained DNA methylation alterations in the sperm of Addict FO and F1 rats. (a) The average methylation levels of CpG sites upstream of the transcription start site (TSS), within the gene body, and the downstream of transcription termination sites (TES) in all genes detected. Addict FO and Non-addict FO showed lower global methylation level than Saline FO. (b) Euclidean distance-based multidimensional scaling indicated segregation of generation and addiction state. Number in circle refers to subject number in each group, corresponding to the subject number in $\mathbf{c}$ (c) Methylation in 1,244 CpGs was significantly different between Addict FO and Non-addict FO, and 522 differentially methylated CpGs were detected in Addict F1 versus Non-addict F1. The differential methylation was maintained in $475 \mathrm{CpGs}$ across F0 to F1 generation. FDR $\leq 0.05$ was considered significant. (d) CpG distributions in TSS $\pm 2,000$ bp area, exons, introns, and other intergenic elements. CpG methylation differences maintained from FO to F1 were enriched in TSS $\pm 2,000$ bp area. Differences maintained from FO to F1/Global, odds ratio (95\% confidence intervals), 273.95 (55.82-1340.73). (e) Ontology analysis of $\mathrm{CpGs}$ (in TSS $\pm 2,000$ bp region) with differential methylation maintained from F0 to F1. Z scores from Enrichr were acquired and ranked. (f) Motif enrichment analysis of TSS $\pm 2,000$ bp region by rGADEM and MotIV. The results revealed specific enrichment of KLF4 binding sites $\left(e=5.67 \times 10^{-10}\right) .(\mathbf{g})$ Methylation profiles of representative $\mathrm{CpGs}$ within differential methylation region (DMR) of KIf4-regulated genes. DMRs were calculated by BiSeq. Group averages are plotted. Btg2: DMR, Chr13: 55970169-55970230, three-way RM ANOVA, $F_{\text {addict } \times \text { generation } \times \text { site }}(9,81)=7.863$, $P=3.03 \times 10^{-8}$; Bonferroni post hoc, $P=3.5 \times 10^{-14}$, Non-addict versus Addict. Nr4a1: DMR, Chr7: 140712984-140713087; three-way RM ANOVA, $F_{\text {addict } \times \text { generation } \times \text { site }}(8,72)=0.832, P=0.58$; Bonferroni post hoc, $P=1.1 \times 10^{-11}$, Non-addict versus Addict. Non-addict F0, $n=3$; Addict F0, $n=3$; Non-addict F1, $n=4$; Addict F1, $n=3$. 
groups (Fig. 8g). This result was consistent with previous report that both Btg2 and Nr4a1 regulate neurogenesis and other brain functions via transcriptional mechanisms ${ }^{22,23}$.

To provide insight into potential mechanistic link of sperm DNA methylation changes to descendant brain functions, we performed transcriptome sequencing of Non-addict F1 and Addict F1 in the nucleus accumbens (NAc). 292 genes exhibit higher expression level in Non-addict F1 than Addict F1 (fold change $\geq 2, P<0.05$ ), around 3.5 -fold of the number downregulated, coincides with lower DNA methylation level in sperms of Non-addict groups than Addict groups (Supplementary Fig. 4a). Although it is well known that DNA methylation profiles change dynamically during development, and differs from sperm to brain, we found that differential promoter methylation in F1 sperm significantly collapsed with alterations of gene expression in adult NAc (Supplementary Fig. 4b). Forty-nine of the 292 genes, namely Gabrd,Chat,Kcnn3 showed transcriptional differences in F1 of Addict versus Non-addict groups, consistent with DNA methylation differences in sperm maintained from F0 to F1 (Supplementary Figs. 4c, d). The differentially methylated and expressed genes from RRBS and RNA-sequencing (RNA-seq) data sets were compared, and a functionally grouped annotation network was deduced. As shown in Supplementary Fig. 4e, genes that differential methylated between Addict and Non-addict groups were enriched in functions that regulate developmental process, consistent with the result shown in Fig. 8e. Several pathways, such as EGFR regulation, gap junction, and axonal guidance pathways, were co-enriched in methylation and transcription data, but in a co-clustering pathway, often distinct genes from methylation and transcription datasets were involved. Network analysis of genes from these clusters reveals possible signalling network connections between genes enriched in methylation and expression. As shown in Supplementary Fig. 4f, ephrins and EPH-related receptors (EPHA5, EFNA1), SHH, ISL1 and TBX5, which strictly controls embryonic development and were differentially methylated in sperm, regulate key plasticity-regulators, such as BDNF, JUN, and Tubulin family members. These downstream molecules were enriched in transcriptome data.

\section{Discussion}

Familial aggregation of drug addiction has been implicated by epidemiological studies ${ }^{10,11}$. Extensive studies have been focused on the identification of genetic variations that are associated with risk of developing drug addiction, and genome-wide linkage and association studies have revealed many genes and genetic variants associated with drug response and related behaviour ${ }^{24-27}$. Significant intergenerational effects of maternal substance exposure during pregnancy on the development and behaviour of offspring have been reported in many experimental studies. Epidemiological data suggests attention deficit, hyperactivity disorder and learning disabilities ${ }^{28}$ in children with prenatal cocaine experience. Enhanced cocaine self-administration ${ }^{29}$, blunted drug sensitivity ${ }^{30}$ and developmental delays ${ }^{31}$ were observed in animal models. Intergenerational effects of prior cocaine experience transmitted via germline have received recent attention. It was reported that maternal cocaine exposure before pregnancy can serve to enhance psychomotor sensitivity to cocaine in offspring ${ }^{32}$. Paternal cocaine use causes intergenerational cocaine-resistant phenotype, anxiety ${ }^{33}$, depression-like behaviour ${ }^{34}$, impaired attention and working memory ${ }^{35}$. Experimentation is still needed to explain the apparent contrast of subtle behaviour changes in animal models with the high liability in human epidemiological studies. ${ }^{36}$ Studies supporting non-Mendelian heritable factors that may predispose offspring to drug addiction are lacking. In the current study, we used a rat model of cocaine self-administration and showed that high incentive motivation elicited by voluntary cocaine administration could be transmitted to subsequent generations. We also show that the transgenerational inheritance effect is not caused by pre-existing genetic variations, but rather it is an acquired trait that is induced by highly motivated drug-seeking experience.

Furthermore, our data indicated that the effect of noncontingent drug intake and reinforcing properties of cocaine on offspring are distinct. Findings by Vassoler et al. ${ }^{19}$, together with the present data, demonstrate that the paternal exposure to cocaine results in reduced cocaine seeking behaviour. Drug exposure per se acts as a novel chemical exposure, leading to protective or deleterious effects in offspring in some addictionresilient individuals, such as drug-resistance ${ }^{19}$, increased anxiety level $^{34}$ and impaired learning and memory ${ }^{37}$, raising concerns on the potential adverse effects of using psychostimulant, and perhaps other drugs. Moreover, we found that it is high motivation for cocaine reinforcement elicited by voluntary drug self-administration, not the total intake of addictive drug itself, that contributes to transgenerational inheritance of high incentive motivation for cocaine, implicating a key role of high responding to incentive reward induced by addictive drugs in the transgenerational inheritance. Our results suggest that highly motivated drug seeking experience may increase the vulnerability to drug reinforcement in descendants, and/or decrease resistance to drug addiction. It is possible that under certain circumstance, the decrease in resistance to drug addiction or absence of protection, may render offspring of Addict animals more vulnerable to cocaine addiction.

Emerging evidence suggests that epigenetic mechanisms, regulated by environmental stimuli, allow individuals to adapt to the dynamic changes in the surrounding environment ${ }^{38-40}$, including changes in nutritional and emotional status ${ }^{41,42}$. Studies indicate that gamete epigenomic information, including DNA methylation, chromatin patterning, noncoding RNAs and mitochondria, is vital to transgenerational inheritance of acquired phenotypes $^{20}$. In the present study, we observed segregation in DNA methylation patterns in the sperm of Addict and Nonaddict F0 rats and their F1 descendants, and identified regions that exhibit maintained differential methylation in F0 and F1, providing an epigenetic link to transcriptomic changes of addiction-related signalling pathways in offspring NAc. Our study demonstrated that highly motivated cocaine-seeking experience induced DNA methylation alterations different from that induced by the intake of cocaine itself, and such epigenetic alterations could be transmitted to subsequent generations, in parallel with transgenerational inheritance of high responding to drug reward. These observations support an epigenetic mechanism for the transgenerational inheritance of incentive motivational properties for cocaine, which includes, but is not limited to DNA methylation. Although the contribution of sperm DNA methylation to descendant neuronal function is not clear, we speculate that so-called epigenetic engrams ${ }^{43}$ of addictionlike behavioural experience in previous generations may induce adaptation of brain functions in offspring to facilitate prompt and favourable adaptive responses on their exposure to cocaine. However, factors that contribute to individual variabilities of motivational response to cocaine remain to be understood.

\section{Methods}

Animals and housing. All experimental naive Sprague-Dawley rats (Shanghai Center of Experimental Animal, Chinese Academy of Sciences) and their descendants were housed at $23^{\circ} \mathrm{C}$ with a $12 \mathrm{~h}$ reverse dark/light cycle (on 20:00 hours, off 08:00 hours). They were allowed free access to food and water before behavioural 
experiments and housed in groups (3-4 rats per cage) before surgery. Room humidity was controlled at $40 \%$. All the behavioural experiments were carried on using male rats aged 8-12 weeks. F0 rats were habituated for at least 2 weeks before experimentation. All animal treatments were strictly in accordance with the National Institutes of Health Guide for the Care and Use of Laboratory Animals and were approved by Animal Care and Use Committee of Shanghai Medical College of Fudan University.

Food training and surgery. To facilitate the rats to self-administer cocaine, rat $8-10$ weeks old were food-restricted and maintained at $85 \%$ of their ad libitum body weight 3 days before, and were then trained to press the active lever to get 45 $\mathrm{mg}$ food pellets in the operant chambers (Med-Associates) for 7 days. The training procedure was FR1 (one lever press/pellet) for 5 days and then FR5 (five lever presses/pellet) for 2 days. The rats which met the criteria of obtaining 100 food pellets per FR5 session were subjected to surgery. These rats were anaesthetized with chloral hydrate $\left(0.4 \mathrm{~g} \mathrm{~kg}^{-1}\right.$, Sangon) intraperitoneally. We positioned a silastic catheter about $3 \mathrm{~cm}$ into the right jugular vein and attached the other end with a stainless steel pedestal. The pedestal was then mounted to the rat's skull by dental cement. The rats were allowed to recover for 7 days after surgery. The catheters were flushed daily with $0.1 \mathrm{ml}$ saline containing heparin $\left(30 \mathrm{IU} \mathrm{ml}^{-1}\right)$ and gentamycin $\left(0.5 \mathrm{mg} \mathrm{ml}^{-1}\right)$.

Intravenous cocaine self-administration. After recovery, rats were allowed to self-administer cocaine in a daily $4 \mathrm{~h}$ FR session. When the rat presses the active press, an injection of cocaine (Qinghai Pharmaceutical Firm) at $0.75 \mathrm{mg} \mathrm{kg}^{-1}$ per injection over $4 \mathrm{~s}$ was presented and accompanied by a conditioned cue, including the illumination of the stimulus light and an audible tone for $20 \mathrm{~s}$. The press on the inactive lever had no programmed consequences. In our procedure, the rats were first trained under the FR1 program for 5 days, then FR3 for 2 days and FR5 for 5 days. Then the program was changed to a $6 \mathrm{~h}$ progressive-ratio (PR) schedule, in which response requirements for each successive injection increased by progressive increments. The response requirement for each injection (i) follows

$\mathrm{i}^{\mathrm{th}_{\text {injection }}}=\operatorname{Int}\left(5 \mathrm{e}^{0.25 \mathrm{i}-5}\right)$ and the session stops when the rat takes $>1 \mathrm{~h}$ to satisfy the response requirement. The number of lever presses for the last injection in the PR schedule was defined as the break point. The catheter patency was verified after PR schedule by anaesthesia with chloral hydrate and the data of rats with catheter problem was excluded. Lever presses during FR5 and break point of individual rats were scored $(\mathrm{x})$ as before ${ }^{44}$, following $X=\frac{\left(X_{\mathrm{i}}-\bar{X}\right)}{\text { s.d. }} . X_{\mathrm{i}}$ is the behaviour value for each rats, $\bar{X}$ is the mean behaviour value for all the rats, and s.d. is the s.d. of the population behaviour value. Twenty-four hours after the last self-administration session, each male rat was housed with two naive female rats to generate F1. F2 rats were sired by crossing naive $\mathrm{F} 1$ with two naive female rats.

Sucrose and food self-administration. The rats were initially maintained at $85 \%$ original body weight for 3 days before the training and trained to press the active lever to get a $45 \mathrm{mg}$ sucrose pellet or grain-based food pellet (Bio-Serv) under FR1 program for 5 days, then FR5 for 2 days and switched to PR schedule.

Dose-response effect on locomotor activity. Rats were habituated to the locomotor chamber for one hour, and were then administered hourly ascending dose of cocaine $\left(0,2.5,7.5,15,25 \mathrm{mg} \mathrm{kg}^{-1}\right.$, intraperitoneal). Locomotor activity was measured during each hour of the testing period.

Open field test. Locomotor activity was measured using commercial open field activity chambers for rats (Med-Associates). The session lasted for $30 \mathrm{~min}$. Data collection and analysis were done using Med Associates Activity Monitor program.

Cocaine intraperitoneal injection. Naive male SD rats were injected intraperitoneally with cocaine $\left(15 \mathrm{mg} \mathrm{kg}^{-1}\right)$ or equivalent volume of saline every $12 \mathrm{~h}$ for 7 days, and were immediately returned to their home cage after each injection. Twenty-four hours after the last injection, each male rat was housed with two naive female rats to generate F1. F2 rats were sired by crossing naive F1 with two naive female rats.

Sperm collection and purification. Male rats were euthanized with chlorohydrate overdose. The testes and cauda epidymides were dissected, washed with PBS and placed in $35 \mathrm{~mm}$ petri dishes containing HBSS supplemented with $20 \mathrm{mM}$ HEPES pH $7.2,1.2 \mathrm{mM} \mathrm{MgSO}_{4} \cdot 7 \mathrm{H}_{2} \mathrm{O}, 1.3 \mathrm{mM} \mathrm{CaCl} 2 \cdot 2 \mathrm{H}_{2} \mathrm{O}, 6.6 \mathrm{mM}$ sodium pyruvate, $0.05 \%$ lactate, $2 \mathrm{mM}$ GlutaMAX Supplement (Gibco) and $0.5 \% \mathrm{BSA}^{45}$. Released sperm-containing supernatant was moved to $5 \mathrm{ml}$ collection tubes and let stand for $10 \mathrm{~min}$ at $37^{\circ} \mathrm{C}$ for swimming up. Supernatant was further filtered through $40 \mu \mathrm{m}$ nylon mesh and laid on top of a three-layer gradient of 80,60 and 30\% Percol, centrifuged for $10 \mathrm{~min}$ at $1,000 \mathrm{~g}$ at room temperature. Sperms were collected from $60 \%$ layer of the gradient, washed with the incubation buffer and cryopreserved. DNA was extracted from purified sperm according to the protocol described by Griffin $^{46}$ and then preserved in TE buffer.
Reduced representation bisulfite sequencing. Library preparation was performed by BasePair BioTechnology. The quality and integrity of DNA samples were controlled by A260/280 ratio ( $\geq 1.8$ ) and agarose gel electrophoresis. For library preparation, $1 \mu \mathrm{g}$ genomic DNA was digested by MspI to generate short fragments that contain $\mathrm{CpG}$ dinucleotides at the ends. Then digested DNA was end-repaired, A-tailed and ligated to methylated Illumina adapters following the user manual of Truseq DNA Sample Prep Kit (Illumina, USA) Then, the CpG-rich DNA fragments (160-340 bp) are size selected, subjected to bisulfite conversion by MethylCode Bisulfite Conversion Kit (Life Technologies, USA), and PCR amplified by ZymoTaq PreMix (Zymo, USA). The libraries were sequenced $125 \mathrm{bp}$ PE on Illumina Hiseq 2500. At least three biological replicates were sequenced for each condition.

RNA sequencing. Total RNA was extracted from NAc of drug- and experimentnaive Addict and Non-addict F1 rats with standard Trizol method. RNA from three rats per group was pooled and subjected to RNA integrity analysis and subsequent library preparation procedures with TruSeq RNA Library Prep Kit (Illumina, USA). 100-bp PE reads were generated with Illumina Hiseq 2000.

Sequencing data analysis. The raw RRBS sequencing reads were quality checked and trimmed with Trim Galore (http://www.bioinformatics.babraham.ac.uk/projects/trim_galore/) for low quality (Phred score $<30$ ) nucleotides, adapters and artificially introduced bases in the end-repair step. Reads too short $(<20 \mathrm{bp})$ were filtered. Alignment and methylation calling of the filtered reads were then performed with Bismark ${ }^{47}$ using genome assembly for Rattus norvegicus UCSC (rn5, RefSeq gene annotations). Parameters used were default except that 3 maximum mismatches in the 'seed' were allowed and 'paired-end' was specified. Bowtie $^{48}$ short read aligner was used. The bisulfite conversion rate was estimated by calculating the $\mathrm{C}$ to $\mathrm{T}$ conversion at non-CpG sites. Differential methylation region was calculated with BiSeq ${ }^{49}$. DMRs were considered significant with $P<0.05$. rGADEM ${ }^{50}$ was used for unseeded motif analysis with default settings and MotIV for e value calculation and visualization based on JASPAR 2010 scores. Euclidean-based multidimensional scaling and DNA methylation profiles across genes were plotted with RnBeads ${ }^{51}$. Expression in heatmap were clustered based on Euclidean distances with R package 'pheatmap'. RNA-seq data was aligned to rn5 and calculated for differential expression with the Tuxedo suite $\mathrm{e}^{52,53}$ using default settings. ClueGO was used to construct network relationships of genes and pathways from RRBS and RNA-seq data set ${ }^{54}$.

Western blotting. Drug- and experiment- naive F1 rats from Saline, Non-addictSA, Non-addict-Yoke, Addict-SA and Addict-Yoke groups were killed. Brains were dissected immediately, and $\mathrm{mPFC}$ was collected and homogenized on ice in $200 \mu \mathrm{l}$ of lysis buffer $(10 \mathrm{mM}$ Tris $\cdot \mathrm{HCl}, \mathrm{pH} 7.4,0.5 \%$ Triton X-100, 0.5\% Nonidet P-40, $50 \mathrm{mM} \mathrm{NaCl}, 1.0 \mathrm{mM}$ EDTA, $50 \mathrm{mM} \mathrm{NaF}, 100 \mu \mathrm{M} \mathrm{Na} \mathrm{VO}_{4}, 1.0 \mu \mathrm{M}$ PMSF and protease inhibitors). After 30-min incubation on ice, lysate was centrifuged at $16,000 \times \mathrm{g}$ for $10 \mathrm{~min}$ and supernatant was collected. Bradford assay was used for protein level normalization. Samples were boiled in SDS/PAGE loading buffer $(0.1 \mathrm{mM}$ Tris $\cdot \mathrm{HCl}, \mathrm{pH} 6.8,5 \%$ glycerol, $1.5 \%$ SDS, $5 \% \beta$-mercaptoethanol, and $0.002 \%$ bromophenol blue) for $5 \mathrm{~min}$. For each sample, $30 \mu \mathrm{g}$ total protein was loaded and separated by $18 \%$ SDS-PAGE. Blots were probed with primary antibody at $4{ }^{\circ} \mathrm{C}$ overnight: anti-BDNF (ab6201, Abcam, $1: 100$ ), and anti- $\beta$-actin (A2066, Sigma-Aldrich, 1:2,000). Blots were then incubated with IRDye $800 \mathrm{CW}$ conjugated or $700 \mathrm{CW}$-conjugated secondary antibodies (Rockland Biosciences). Infrared fluorescence images were obtained with the Odyssey infrared imaging system (Li-Cor Bioscience). Quantification was performed with Image Studio Lite Ver 5.2. The blotting experiments were repeated three times and average expression of each sample was used. BDNF level was normalized by comparing with the coordinate $\beta$-Actin expression level. Uncropped image for the representative plot is provided in Supplementary Fig. 5.

Statistical analysis. Behavioural data in cocaine self-administration, sucrose selfadministration during FR program was analysed with mixed linear model with repeated measurements (MMRM). Cocaine dose induced locomotion were analysed using two-way repeated-measures analysis of variance (ANOVA) followed by Bonferroni post hoc tests, break point was analysed by Mann-Whitney Rank-Sum Test or Friedman two-way ANOVA test by ranks. The open field test and real-time PCR data were analysed by Student's $t$ test. Sample size estimation was conducted on alpha value of 0.05 and desired power of $0.80 . P<0.05$ was considered statistically significant. Data are presented as mean \pm s.e.m.

Data availability. Raw sequencing data that support the findings of this study have been deposited in the Gene Expression Ominibus with accession code GSE72401 (http://www.ncbi.nlm.nih.gov/geo/query/acc.cgi?acc=GSE72401). The authors declare that all other data supporting the findings of this study are available within the manuscript and its supplementary files or are available from the corresponding author on request. 


\section{References}

1. Stewart, J. Evolution's Arrow: The Direction of Evolution and the Future of Humanity (Chapman Press, 2000).

2. Daxinger, L. \& Whitelaw, E. Understanding transgenerational epigenetic inheritance via the gametes in mammals. Nat. Rev. Genet. 13, 153-162 (2012).

3. Bohacek, J., Gapp, K., Saab, B. J. \& Mansuy, I. M. Transgenerational epigenetic effects on brain functions. Biol. Psychiatry 73, 313-320 (2013).

4. Bale, T. L. Epigenetic and transgenerational reprogramming of brain development. Nat. Rev. Neurosci. 16, 332-344 (2015).

5. Anway, M. D., Cupp, A. S., Uzumcu, M. \& Skinner, M. K. Epigenetic transgenerational actions of endocrine disruptors and male fertility. Science 308, 1466-1469 (2005).

6. Dunn, G. A. \& Bale, T. L. Maternal high-fat diet effects on third-generation female body size via the paternal lineage. Endocrinology 152, 2228-2236 (2011).

7. Cami, J. \& Farre, M. Drug addiction. N. Engl. J. Med. 349, 975-986 (2003).

8. Robison, A. J. \& Nestler, E. J. Transcriptional and epigenetic mechanisms of addiction. Nat. Rev. Neurosci. 12, 623-637 (2011).

9. Hyman, S. E., Malenka, R. C. \& Nestler, E. J. Neural mechanisms of addiction: the role of reward-related learning and memory. Annu. Rev. Neurosci. 29, 565-598 (2006).

10. Merikangas, K. R. et al. Familial transmission of substance use disorders. Arch. Gen. Psychiatry 55, 973-979 (1998).

11. Bierut, L. J. et al. Familial transmission of substance dependence: alcohol, marijuana, cocaine, and habitual smoking: a report from the collaborative study on the genetics of alcoholism. Arch. Gen. Psychiatry 55, 982-988 (1998).

12. Treutlein, J. \& Rietschel, M. Genome-wide association studies of alcohol dependence and substance use disorders. Curr. Psychiatry Rep. 13, 147-155 (2011).

13. Kendler, K. S., Prescott, C. A., Myers, J. \& Neale, M. C. The structure of genetic and environmental risk factors for common psychiatric and substance use disorders in men and women. Arch. Gen. Psychiatry 60, 929-937 (2003).

14. Tsuang, M. T. et al. Co-occurrence of abuse of different drugs in men: the role of drug-specific and shared vulnerabilities. Arch. Gen. Psychiatry 55, 967-972 (1998).

15. Li, M. D. The genetics of nicotine dependence. Curr. Psychiatry Rep. 8, 158-164 (2006).

16. Goldman, D., Oroszi, G. \& Ducci, F. The genetics of addictions: uncovering the genes. Nat. Rev. Genet. 6, 521-532 (2005).

17. Anthony, J. C., Warner, L. A. \& Kessler, R. C. Comparative epidemiology of dependence on tobacco, alcohol, controlled substances, and inhalants: basic findings from the national comorbidity survey. Exp. Clin. Psychopharmacol. 2, 244-268 (1994)

18. Deroche-Gamonet, V., Belin, D. \& Piazza, P. V. Evidence for addiction-like behavior in the rat. Science 305, 1014-1017 (2004).

19. Vassoler, F. M., White, S. L., Schmidt, H. D., Sadri-Vakili, G. \& Pierce, R. C. Epigenetic inheritance of a cocaine-resistance phenotype. Nat. Neurosci. 16, 42-47 (2013).

20. Lane, M., Robker, R. L. \& Robertson, S. A. Parenting from before conception. Science 345, 756-760 (2014)

21. Hu, S. et al. DNA methylation presents distinct binding sites for human transcription factors. Elife 2, e00726 (2013).

22. Chen, Y. et al. Activity-induced Nr4al regulates spine density and distribution pattern of excitatory synapses in pyramidal neurons. Neuron 83, 431-443 (2014).

23. Calegari, F., Haubensak, W., Haffner, C. \& Huttner, W. B. Selective lengthening of the cell cycle in the neurogenic subpopulation of neural progenitor cells during mouse brain development. J. Neurosci. 25, 6533-6538 (2005)

24. Zhang, D. et al. Effect of mu-opioid receptor gene polymorphisms on heroininduced subjective responses in a Chinese population. Biol. Psychiatry 61, 1244-1251 (2007)

25. Sadler, B. et al. Variants near Chrnb3-Chrna6 are associated with Dsm-5 cocaine use disorder: evidence for pleiotropy. Sci. Rep. 4, 4497 (2014).

26. Weiss, R. B. et al. A candidate gene approach identifies the Chrna5-A3-B4 region as a risk factor for age-dependent nicotine addiction. PLoS Genet. 4, e1000125 (2008)

27. Nielsen, D. A., Utrankar, A., Reyes, J. A., Simons, D. D. \& Kosten, T. R. Epigenetics of drug abuse: predisposition or response. Pharmacogenomics 13, 1149-1160 (2012)

28. Mayes, L., Snyder, P. J., Langlois, E. \& Hunter, N. Visuospatial working memory in school-aged children exposed in utero to cocaine. Child Neuropsychol. 13, 205-218 (2007).

29. Keller, Jr R. W., LeFevre, R., Raucci, J., Carlson, J. N. \& Glick, S. D. Enhanced cocaine self-administration in adult rats prenatally exposed to cocaine. Neurosci. Lett. 205, 153-156 (1996).

30. Crozatier, C. et al. Altered cocaine-induced behavioral sensitization in adult mice exposed to cocaine in utero. Brain Res. Dev. Brain Res. 147, 97-105 (2003).

31. Bellone, C., Mameli, M. \& Luscher, C. In utero exposure to cocaine delays postnatal synaptic maturation of glutamatergic transmission in the Vta. Nat. Neurosci. 14, 1439-1446 (2011)
32. Sasaki, A. et al. Cocaine exposure prior to pregnancy alters the psychomotor response to cocaine and transcriptional regulation of the dopamine D1 receptor in adult male offspring. Behav. Brain Res. 265, 163-170 (2014)

33. White, S. L., Vassoler, F. M., Schmidt, H. D., Pierce, R. C. \& Wimmer, M. E. Enhanced anxiety in the male offspring of sires that self-administered cocaine. Addict. Biol. 21, 802-810 (2015).

34. Killinger, C. E., Robinson, S. \& Stanwood, G. D. Subtle biobehavioral effects produced by paternal cocaine exposure. Synapse 66, 902-908 (2012).

35. He, F., Lidow, I. A. \& Lidow, M. S. Consequences of paternal cocaine exposure in mice. Neurotoxicol. Teratol. 28, 198-209 (2006).

36. Singh, D. P. et al. Genome-defence small rnas exapted for epigenetic matingtype inheritance. Nature 509, 447-452 (2014)

37. Yohn, N. L., Bartolomei, M. S. \& Blendy, J. A. Multigenerational and transgenerational inheritance of drug exposure: the effects of alcohol, opiates, cocaine, marijuana, and nicotine. Prog. Biophys. Mol. Biol. 118, 21-33 (2015).

38. Richards, E. J. Inherited epigenetic variation-revisiting soft inheritance. Nat. Rev. Genet. 7, 395-401 (2006).

39. Manikkam, M., Tracey, R., Guerrero-Bosagna, C. \& Skinner, M. K. Plastics derived endocrine disruptors (Bpa, Dehp and Dbp) induce epigenetic transgenerational inheritance of obesity, reproductive disease and sperm epimutations. PLoS ONE 8, e55387 (2013).

40. Skinner, M. K., Manikkam, M. \& Guerrero-Bosagna, C. Epigenetic transgenerational actions of endocrine disruptors. Reprod. Toxicol. 31, 337-343 (2011).

41. Toth, M. Mechanisms of non-genetic inheritance and psychiatric disorders. Neuropsychopharmacology 40, 129-140 (2015).

42. Bohacek, J. \& Mansuy, I. M. Molecular insights into transgenerational nongenetic inheritance of acquired behaviours. Nat. Rev. Genet. 16, 641-652 (2015)

43. Jablonka, E. \& Raz, G. Transgenerational epigenetic inheritance: prevalence, mechanisms, and implications for the study of heredity and evolution. Q Rev. Biol. 84, 131-176 (2009).

44. Bock, R. et al. Strengthening the accumbal indirect pathway promotes resilience to compulsive cocaine use. Nat. Neurosci. 16, 632-638 (2013).

45. Lassalle, B. et al. 'Side Population' cells in adult mouse testis express Bcrpl gene and are enriched in spermatogonia and germinal stem cells. Development 131, 479-487 (2004).

46. Griffin, J. Methods of sperm DNA extraction for genetic and epigenetic studies Methods Mol. Biol. 927, 379-384 (2013).

47. Krueger, F. \& Andrews, S. R. Bismark: a flexible aligner and methylation caller for bisulfite-seq applications. Bioinformatics 27, 1571-1572 (2011).

48. Langmead, B., Trapnell, C., Pop, M. \& Salzberg, S. L. Ultrafast and memoryefficient alignment of short DNA sequences to the human genome. Genome Biol. 10, R25 (2009).

49. Hebestreit, K., Dugas, M. \& Klein, H. U. Detection of significantly differentially methylated regions in targeted bisulfite sequencing data. Bioinformatics 29, 1647-1653 (2013).

50. Droit, A. et al. Discovery, $R$ package version 2.14 .0 edn. Available at: http:// bioconductor.org/packages/rGADEM/ (2014).

51. Assenov, Y. et al. Comprehensive analysis of DNA methylation data with Rnbeads. Nat. Methods 11, 1138-1140 (2014).

52. Trapnell, C. et al. Differential gene and transcript expression analysis of RNAseq experiments with tophat and cufflinks. Nat. Protoc. 7, 562-578 (2012).

53. Mortazavi, A., Williams, B. A., McCue, K., Schaeffer, L. \& Wold, B. Mapping and quantifying mammalian transcriptomes by RNA-seq. Nat. Methods 5, 621-628 (2008)

54. Bindea, G. et al. Cluego: a cytoscape plug-in to decipher functionally grouped gene ontology and pathway annotation networks. Bioinformatics 25, 1091-1093 (2009).

\section{Acknowledgements}

We thank Dr Nashat Abumaria from Institutes of Brain Science, Fudan University, for critical reading of the manuscript, and Dr Naiqing Zhao from School of Public Health, Fudan University, for statistical assistance. This work was supported by the Ministry of Science and Technology (2015CB553500, 2014CB942801 and 2013CB835102) and the National Natural Science Foundation of China (31430033, 91232307, 31421091 and 81272295).

\section{Author contributions}

B.Y., Y.L., Q.L., X.Y., H.S., H.Z., W.H. and Y.Z. carried out experiments. B.Y. and Q.L. performed statistical analysis, Q.L., D.M., F.W., analysed sequencing data. L.M., B.Y. and Q.L. designed the study and wrote the manuscript X.Y. and Y.L. contributed equally to this work.

\section{Additional information}

Supplementary Information accompanies this paper at http://www.nature.com/ naturecommunications

Competing interests: The authors declare no competing financial interests. 
Reprints and permission information is available online at http://npg.nature.com/ reprintsandpermissions/

How to cite this article: Le, Q. et al. Drug-seeking motivation level in male rats determines offspring susceptibility or resistance to cocaine-seeking behaviour. Nat. Commun. 8, 15527 doi: 10.1038/ncomms15527 (2017).

Publisher's note: Springer Nature remains neutral with regard to jurisdictional claims in published maps and institutional affiliations. (c) (i) This work is licensed under a Creative Commons Attribution 4.0 International License. The images or other third party material in this article are included in the article's Creative Commons license, unless indicated otherwise in the credit line; if the material is not included under the Creative Commons license, users will need to obtain permission from the license holder to reproduce the material. To view a copy of this license, visit http://creativecommons.org/licenses/by/4.0/

(C) The Author(s) 2017 